\title{
NOTE ON PRESERVATION OF MEASURABILITY
}

\section{ROY B. LEIPNIK}

If $m$ measures $X$ (that is, $m$ is a countably subadditive function on subsets of $X$ to the non-negative reals), and if $f$ is a function on subsets of $Y$ to subsets of $X$, it is natural to inquire into the behavior of the composite function $n$, where $n(A)=m(f(A))$ for each $A \subset Y$. Rather obvious sufficient conditions are given below for $n$ to measure $Y$, and for a subset $A$ of $Y$ to be $n$-measurable.

Several definitions are necessary. A subset $C$ of $X$ is called $m$-measurable- $F$ if and only if $m(T \cap D)=m(T \cap D \cap C)+m(T \cap D \cap(X-C))$ whenever $T \subset X$ and $D \in F$. A subset $B$ of $Y$ is called a set of $m$-continuity- $f$ if and only if $m(f(B) \cap f(Y-B))=0$. Suppose henceforth that $\left(^{*}\right) m\left(f(A) \cap\left(X-\cup_{B \in \mathcal{C}} f(B)\right)\right)=0$ whenever $A \subset Y$ and $\mathfrak{H C}$ is a countable covering of $A$ by subsets of $Y$.

Theorem 1. If $\left({ }^{*}\right)$, then $n$ measures $Y$.

Proof. If $A \subset Y$, and $\mathfrak{H C}$ is a countable covering of $A$ by

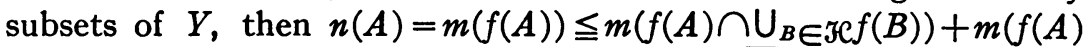
$\left.\cap\left(X-\cup_{B \in \mathcal{F C}} f(B)\right)\right)=m\left(\cup_{B \in \mathcal{F}}(f(A) \cap f(B))\right) \leqq \sum_{B \in \mathcal{H C}}(f(A) \cap f(B))$ $\leqq \sum_{B \in \mathcal{F C}}(f(B))=\sum_{B \in \mathcal{F C} n}(B)$, so that $n$ measures $Y$.

Lemma 1. If $\left({ }^{*}\right), B \subset Y$, and $C \subset Y$, then $n(B \cap C) \leqq m(f(B) \cap f(C))$.

Proof. Since $B \cap C \subset B$ and $B \cap C \subset C, m(f(B \cap C) \cap(X-f(B)$ $\cap f(C)))=m(f(B \cap C) \cap(X-f(B))) \cup(f(B \cap C) \cap(X-f(C)))$ $\leqq m(f(B \cap C) \cap(X-f(B)))+m(f(B \cap C) \cap(X-f(C)))=0$. Hence $n(B \cap C) \leqq m(f(B \cap C) \cap(f(B) \cap f(C)))+m(f(B \cap C) \cap(X-f(B)$ $\cap f(C))) \leqq m(f(B) \cap f(C))$.

TheOREM 2. If $\left({ }^{*}\right), A$ is a set of m-continuity-f, and $f(A)$ is m-measurable-range $f$, then $A$ is n-measurable.

Proof. Suppose $T \subset Y$. By Lemma 1, $n(T \cap A) \leqq m(f(T) \cap f(A))$ and $n(T \cap(Y-A)) \leqq m(f(T) \cap f(Y-A)) \leqq m(f(T) \cap f(Y-A) \cap f(A))$ $+m(f(T) \cap f(Y-A) \cap(X-f(A))) \leqq m(f(A) \cap f(Y-A))+m(f(T)$ $\cap(X-f(A)))$. By hypothesis, $m(f(A) \cap f(Y-A))=0$ and $m(f(T)$ $\cap f(A))+m(f(T) \cap(X-f(A)))=m(f(T))=n(T)$. Therefore $n(T \cap A)$ $+n(T \cap(Y-A)) \leqq n(T)$.

\section{REFERENCE}

1. T. J. McMinn, Restricted measurability, Bull. Amer. Soc. vol. 54 (1948) p. 1106. InSTITUTE FOR AdVANCEd Study

Received by the editors August 5, 1949. 